\title{
Fatores de risco associados à violência contra pessoas idosas na atualidade
}

\author{
Risk factors associated with violence Against older people nowadays
}

Factores de riesgo asociados a la violencia contra los ancianos en la actualidad

Gabriela Sena Nogueira Fonseca Santos ${ }^{1 *}$, Ana Clara Amorim Noronha1, Lucas Meneses Alverga ${ }^{1}$, Fabiana Medeiros de Brito ${ }^{1}$, Luisiane de Avila Silva ${ }^{1}$, Cesar Augusto de Freitas e Rathke'.

\begin{abstract}
RESUMO
Objetivo: Elucidar os fatores de riscos mais prevalentes, associados à Violência Contra a Pessoa Idosa (VCPI), assim como investigar as estratégias adotadas para a prevenção de tal problemática. Métodos: Tratase de uma revisão integrativa de literatura realizada a partir de artigos científicos publicados nas bases de dados MEDLINE, LILACS e BDENF, presentes na Biblioteca Virtual em Saúde (BVS), nos idiomas português, inglês e espanhol entre os anos de 2017 e 2021 . Após a aplicação dos critérios de inclusão e exclusão, foram selecionados 41 estudos para compor a amostra. Resultados: Mediante análise da amostra, emergiu a categoria "Violência contra a pessoa idosa: fatores de risco predominantes no cenário atual e estratégias adotadas para a prevenção a tal problemática". Considerações finais: Enfatiza-se necessidade de enfrentamento a referida problemática, considerando os fatores de risco elencados neste estudo, assim como a necessidade de ações que busquem a prevenção da violência contra a pessoa idosa.
\end{abstract}

Palavras-chave: Maus-tratos ao idoso, Fatores de risco, Idosos.

\begin{abstract}
Objective: Elucidate the most prevalent risk factors associated with Violence Against the Elderly (VAE), as well as investigate the strategies adopted to prevent this problem. Methods: This is an integrative literature review based on scientific articles published in the MEDLINE, LILACS and BDENF databases, present in the Virtual Health Library (VHL), in Portuguese, English and Spanish between 2017 and 2021 After applying the inclusion and exclusion criteria, 41 studies were selected to compose the sample. Results: By analyzing the sample, the category "Violence against the elderly: predominant risk factors in the current scenario and strategies adopted to prevent this problem" emerged. Final considerations: It emphasizes the need to face this problem, considering the risk factors listed in this study, as well as the need for actions that seek to prevent violence against the elderly.
\end{abstract}

Keywords: Elder abuse, Risk factors, Aged.

\section{RESUMEN}

Objetivo: Dilucidar los factores de riesgo más prevalentes asociados a la Violencia Contra las Personas Mayores (VCPM), así como investigar las estrategias adoptadas para prevenir este problema. Métodos: Se trata de una revisión integradora de la literatura basada en artículos científicos publicados en las bases de datos MEDLINE, LILACS y BDENF, presentes en la Biblioteca Virtual en Salud (BVS), en portugués, inglés y español entre 2017 y 2021. Tras aplicar los criterios de inclusión y exclusión, Se seleccionaron 41 estudios para componer la muestra. Resultados: Del análisis de la muestra surgió la categoría "Violencia contra las personas mayores: factores de riesgo predominantes en el escenario actual y estrategias adoptadas para prevenir este problema". Consideraciones finales: Enfatiza la necesidad de enfrentar este problema, considerando los factores de riesgo enumerados en este estudio, así como la necesidad de acciones que busquen prevenir la violencia contra las personas mayores.

Palabras clave: Maltrato al anciano, Factores de riesgo, Anciano.

\footnotetext{
${ }^{1}$ Faculdade de Ciências Médicas da Paraíba (FCM-PB), João Pessoa - PB.

*E-mail: gabrielasena13@gmail.com
} 


\section{INTRODUÇÃO}

O envelhecimento populacional designa-se como um processo em nível mundial, sendo caracterizado por fatores específicos. A transição demográfica e epidemiológica é um processo que vem ocorrendo e tem como fatores causais os avanços da medicina e a entrada da mulher no mercado de trabalho, gerando uma diminuição nas taxas de mortalidade e natalidade, sendo o processo de envelhecimento populacional consequência dessa redução (VASCONCELOS AMN e GOMES MMF, 2012).

No que se refere à transição demográfica, uma estimativa realizada pela Organização das Nações Unidas (ONU) em 2017 apontou que existiam 962 milhões de idosos em todo mundo e que, esse valor ficaria duas vezes maior até 2050, e triplicado em 2100 (UNITED NATIONS, 2015). No Brasil, esse crescimento também se dá de forma muito acelerada. Calcula-se que em 2030, 18,6\% da população brasileira terá 60 anos ou mais, e que em 2060, essa porcentagem aumentará para aproximadamente de $33,7 \%$ (INSTITUTO BRASILEIRO DE GEOGRAFIA E ESTATÍSTICA, 2021).

Considerando o processo de envelhecimento humano, ressalta-se que é crescente também o aumento dos casos de violência contra esse grupo. A Organização Mundial de Saúde (OMS), define violência como o uso de força física ou poder contra outros indivíduos, ou a si próprio, podendo causar sofrimento psicológico ou físico, déficit no desenvolvimento ou privação, e até mesmo o óbito. A violência divide-se em vários tipos, entre estes: violência física, que é caracterizada pela utilização da força física, causando dor, ferimentos, incapacidades ou morte; violência sexual, caracterizada por ato sexual ou uma tentativa deste, fazendo uso de violência física ou ameaça; violência psicológica, por ações que causam danos emocionais como humilhação a partir de ofensas verbais ou privação da liberdade; e a violência econômica, que consiste no uso inadequado e não permitido dos bens do idoso (SANTOS MAB, et al., 2020).

As pessoas idosas, devido às alterações fisiológicas decorrentes do processo de envelhecimento, possuem uma maior vulnerabilidade à violência. As perdas em mobilidade, agilidade e capacidades, em geral, repercutem na supressão da autonomia e da independência. Assim, estar dependente de alguém, seja financeiramente, fisicamente ou no aspecto psíquico, os leva a uma maior susceptibilidade à agressão (BARCELOS EM e MADUREIRA MDS, 2013). Vale ressaltar que abandono, negligência e autonegligência também são considerados tipos de violência. $O$ abandono é caracterizado pela falta de cuidados do responsável para com o idoso, não dando o auxílio necessário. Já a negligência revela-se pela recusa ou omissão à prática dos cuidados, seja pelo responsável ou por um serviço. Por fim, a autonegligência consiste na falta de cuidado do próprio idoso consigo (SANTOS MAB, et al., 2020).

Revelam-se como fatores associados à ocorrência da Violência Contra a Pessoa Idosa (VCPI), o nível de dependência, o abuso de álcool ou substâncias químicas, além do estresse por parte do cuidador. Devido a vergonha ou medo, o idoso nega a violência ou diminui o fato, encontrando desculpas para justificar o acontecido. As agressões têm sérias consequências para o indivíduo acometido, como depressão, raiva contra os parentes e a si mesmo, tentativa de suicídio, dificuldade para se comunicar e tomar decisões, doenças físicas, perda de identidade, falta de confiança, mudanças de crenças e valores e dependência de álcool, jogos, drogas e alimentação (BARCELOS EM e MADUREIRA MDS, 2013).

Visando a proteger a integralidade dos idosos e garantir os direitos destes, foram criadas a Política Nacional do Idoso, em 1994, e a Lei No 10.741, de 1을 de outubro de 2003, conhecida como o Estatuto do Idoso, sendo as principais legislações brasileiras voltadas para a pessoa idosa. Com o intuito de reverter a violência contra o idoso, um dos artigos do referido Estatuto tem como pauta a condenação severa desse ato lesivo, além de apontar como um dever de toda a população denunciar tais práticas. No entanto, ainda assim, tem-se muitos casos impunes (MOURA LKB, et al., 2020).

A partir do exposto percebe-se que os resultados empíricos obtidos por meio deste estudo representam subsídios importantes para o planejamento e a implementação de intervenções específicas, suscitando a melhoria da qualidade de vida da população idosa. Salienta-se também a importância do desenvolvimento de pesquisas que venham complementar a prática dos profissionais de saúde, envolvendo a prevenção e a promoção do cuidado em suas dimensões física, social, psíquica e emocional. Sendo assim, o presente estudo teve como objetivo elucidar os fatores de risco associados à VCPI, identificando na produção científica os fatores mais prevalentes, assim como investigar as estratégias adotadas para a prevenção da VCPI. 


\section{MÉTODOS}

Este estudo constitui-se de uma revisão integrativa da literatura com abordagem qualitativa, de natureza descritiva e explicativa, que tem como finalidade analisar, identificar e sintetizar os resultados independentes sobre o mesmo assunto, incentivando assim, uma compreensão ampla e completa do fenômeno estudado. Dessa forma, contribui-se para uma possível reflexão e repercussão benéfica na qualidade dos cuidados oferecidos às pessoas idosas, como também para a concepção de pensamentos críticos necessários à prática diária no âmbito da saúde (SOUZA MTD, et al., 2010).

Diante disso, percebe-se que a revisão integrativa da literatura se integra na Prática Baseada em Evidências (PBE). Contudo, o preparo dessa revisão baseou-se nas seguintes etapas: elaboração da pergunta norteadora; busca na literatura; coleta de dados usando critérios de inclusão e exclusão; análise crítica dos estudos incluídos; discussão dos resultados e apresentação da revisão (SOUZA MTD, et al., 2010). Para guiar o estudo, definiu-se a seguinte pergunta norteadora: Mediante as evidências científicas, quais os principais fatores de risco relacionados à violência contra a pessoa idosa no cenário atual?

A busca dos dados foi realizada entre os meses de março e maio de 2021, nas bases de dados MEDLINE (Medical Literature Analysis and Retrieval System Online), LILACS (Literatura Latino-americana e do Caribe em Ciências da Saúde), BDENF (Base de Dados de Enfermagem), mediante busca através da Biblioteca Virtual em Saúde (BVS). Os Descritores em Ciências da Saúde (Decs) definidos para responder à questão foram: "Idoso", "Aged", "Anciano", "Violência", "Elder Abuse", "Maltrato al Anciano", "Fatores de Risco", "Risk Factors" e "Fatores de Riesgo". Em seguida, procedeu-se à busca dos artigos, manuseando-se os descritores e os interligando por meio do conector AND.

Inicialmente, realizou-se a busca com os descritores em português, inglês e espanhol e foram eleitos os seguintes critérios de inclusão: texto completo, publicação no período de janeiro de 2017 a março de 2021, nos idiomas em inglês, português e espanhol. Foram excluídos os estudos que não atendessem a questão norteadora e aos critérios de inclusão mencionados, além de artigos duplicados ou que não demonstrassem adequadamente o referencial teórico e metodológico e/ou rigor científico e ético.

\section{RESULTADOS}

Foram obtidos inicialmente 2137 artigos na busca e após a aplicação dos filtros mencionados em métodos, restaram 458 estudos no total. Cumpriu-se em seguida a leitura minuciosa de cada título, resumo e artigo na íntegra, para selecionar os artigos que se relacionavam com o objetivo do estudo. Após essa leitura, 41 artigos foram escolhidos, e suas informações organizadas em um instrumento de coleta de dados, contendo: título do artigo, ano de publicação e base de dados. Por fim, foi realizada uma filtragem de 15 artigos para montagem do Quadro 1 (descrição dos 15 principais filtrados dos 41 artigos selecionados na busca, segundo autor/ ano, objetivos, resultado e conclusão). O fluxograma da seleção dos artigos é apresentado a seguir na Figura 1.

Figura 1 - Fluxograma do processo de busca e identificação dos artigos.

\begin{tabular}{|c|c|c|c|}
\hline $\begin{array}{l}\text { Total: } 2137 \\
\text { artigos } \\
\text { encotrados. }\end{array}$ & $\begin{array}{c}458 \\
\text { artigos. }\end{array}$ & $\begin{array}{c}41 \\
\text { artigos }\end{array}$ & $\begin{array}{l}15 \text { artigos } \\
\text { selecionados para } \\
\text { construção do } \\
\text { Quadro } 1 .\end{array}$ \\
\hline $\begin{array}{c}\text { - Disponpivel na íntegra; } \\
\text { Período de janeiro de } \\
2017 \text { a março de } 2021 ; \\
\text { Em inglês, português } \\
\text { ou espanhol. }\end{array}$ & $\begin{array}{l}\text { • Exclusão de } \\
\text { artigos duplicados } \\
\text { ou com fuga do } \\
\text { tema. }\end{array}$ & $\begin{array}{l}\text { •Após leitura } \\
\text { minuciosa de cada } \\
\text { título, resumo e } \\
\text { artigo na íntegra. }\end{array}$ & $\begin{array}{l}\text {-Artigos com } \\
\text { informações } \\
\text { mais relevantes } \\
\text { para o estudo. }\end{array}$ \\
\hline
\end{tabular}

Fonte: Santos GSNF, et al., 2021. 
Quadro 1 - Quadro-síntese com as principais identificações dos estudos.

\begin{tabular}{|c|c|c|c|}
\hline Autor/ ano & Objetivos & Resultado & Conclusão \\
\hline $\begin{array}{l}\text { Makaroun LK, et al. } \\
(2021) .\end{array}$ & $\begin{array}{l}\text { Examinar as mudanças autorrelatadas } \\
\text { nos fatores de risco relacionados aos } \\
\text { maus tratos do cuidador para com idosos } \\
\text { durante a pandemia de COVID- } 19 \text {. }\end{array}$ & $\begin{array}{l}\text { Os cuidadores de idosos entrevistados neste estudo } \\
\text { relataram que a COVID-19 tornou o cuidado mais difícil } \\
\text { fisicamente (18,7\%), emocionalmente (48,5\%) e } \\
\text { financeiramente }(14,5 \%) \text {, aumentando o risco de abuso. }\end{array}$ & $\begin{array}{l}\text { O reconhecimento dos fatores de risco de abuso de idosos } \\
\text { para os cuidadores e como eles estão sendo impactados } \\
\text { pelo COVID-19 pode permitir que os prestadores de } \\
\text { serviços de saúde e serviços sociais avaliem esses fatores } \\
\text { de risco, reduzindo o risco de abuso de idosos durante a } \\
\text { pandemia de COVID-19. }\end{array}$ \\
\hline $\begin{array}{l}\text { Antequera IG, et al. } \\
(2021) .\end{array}$ & 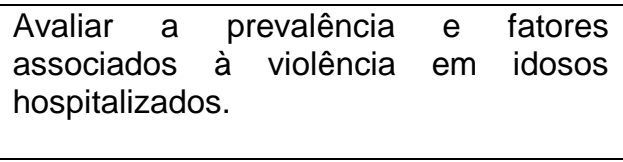 & $\begin{array}{l}\text { Os fatores associados ao risco aumentado para violação } \\
\text { de direitos pessoais ou abuso direto nos idosos foram } \\
\text { maior idade, apresentar estresse percebido e ter } \\
\text { sintomas de depressão leve a severa. }\end{array}$ & $\begin{array}{l}\text { É de extrema importância a observação atenta do idoso } \\
\text { por parte do enfermeiro para permitir a identificação do } \\
\text { risco para violência ou violação de direitos. O que } \\
\text { possibilita estabelecer ações preventivas. }\end{array}$ \\
\hline $\begin{array}{c}\text { Filipska K, et al. } \\
\text { (2020). }\end{array}$ & $\begin{array}{l}\text { Explorar a prevalência, perpetradores e } \\
\text { preditores de abuso psicológico em } \\
\text { idosos. }\end{array}$ & $\begin{array}{l}\text { Quanto menor a renda mensal, maior o risco de violência } \\
\text { psicológica. Os entrevistados mais velhos sofrem } 3 \\
\text { vezes do que as pessoas da faixa etária mais jovem em } \\
\text { relação à violência. Pessoas com doenças crônicas são } \\
\text { estatisticamente mais propensas a sofrer violência na } \\
\text { forma de isolamento }\end{array}$ & $\begin{array}{l}\text { O abuso psicológico é vivenciado por mulheres, idosos } \\
\text { residentes em áreas urbanas e pessoas com baixo nível } \\
\text { socioeconômico e doença crônica. }\end{array}$ \\
\hline $\begin{array}{l}\text { Sudan A, et al. } \\
\qquad(2020) .\end{array}$ & $\begin{array}{l}\text { Avaliar a prevalência de abuso em } \\
\text { idosos com doença psiquiátrica e sua } \\
\text { associação com diversas variáveis } \\
\text { sociodemográficas. }\end{array}$ & $\begin{array}{l}\text { Uma prevalência significativamente maior de abuso foi } \\
\text { observada em idosos com doença psiquiátrica }(21,3 \%) \\
\text { em comparação com aqueles com doença somática } \\
(4 \%) \text {. }\end{array}$ & $\begin{array}{l}\text { O abuso de idosos é um grave problema social. A } \\
\text { conscientização deve ser aumentada para melhorar a } \\
\text { atitude e o comportamento em relação aos idosos. }\end{array}$ \\
\hline $\begin{array}{c}\text { Sembiah S, et al, } \\
(2020) .\end{array}$ & $\begin{array}{l}\text { Revelar a magnitude do abuso de idosos } \\
\text { e seus preditores. }\end{array}$ & $\begin{array}{l}\text { O estudo constatou que } 25,6 \% \text { dos entrevistados } \\
\text { sofreram abuso, sendo o abuso psicológico o tipo mais } \\
\text { comum. A deficiência foi o risco percebido mais comum } \\
\text { para o abuso. }\end{array}$ & $\begin{array}{l}\text { A triagem de rotina para o abuso de idosos e as } \\
\text { campanhas de prevenção de abuso podem ser uma } \\
\text { medida importante para aliviar esse problema. }\end{array}$ \\
\hline $\begin{array}{c}\text { Makaroun LK, et al. } \\
\text { (2020). }\end{array}$ & $\begin{array}{l}\text { Esclarecer, do ponto de vista de } \\
\text { profissionais de saúde, os impactos da } \\
\text { COVID-19 em pessoas mais velhas e em } \\
\text { seus cuidadores, correlacionando aos } \\
\text { riscos de abuso de idosos durante a } \\
\text { pandemia de COVID-19. }\end{array}$ & $\begin{array}{l}\text { Para os idosos, o isolamento social é um fator de risco } \\
\text { conhecido para sofrer abuso, os limites de contato } \\
\text { pessoal podem limitar muito a capacidade dos } \\
\text { cuidadores de prestar atendimento pessoalmente e, em } \\
\text { geral, limitar as oportunidades para que o abuso } \\
\text { contínuo de idosos seja detectado por outras pessoas. }\end{array}$ & $\begin{array}{l}\text { Embora a presença do cuidador possa trazer benefícios, } \\
\text { também pode dificultar a divulgação de abuso ou } \\
\text { negligência, se presente. Então, profissionais de saúde, } \\
\text { para mitigar a VCPI, devem observar sistematicamente os } \\
\text { sinais de situações inseguras e indagar diretamente sobre } \\
\text { a segurança e o bem-estar dos idosos. }\end{array}$ \\
\hline $\begin{array}{c}\text { Koga C, et al. } \\
\text { (2020). }\end{array}$ & $\begin{array}{l}\text { Esclarecer a prevalência e os fatores } \\
\text { associados ao abuso de idosos entre } \\
\text { idosos independentes no Japão. }\end{array}$ & $\begin{array}{l}\text { Os fatores associados ao aumento das chances de } \\
\text { sofrer abuso foram ser mulher, morar com familiares, ter } \\
\text { autoavaliação de saúde ruim e ter depressão leve ou } \\
\text { grave. Por outro lado, idade } \geq 85 \text { anos, ser viúvo ou } \\
\text { solteiro e ter uma visão positiva da confiança da } \\
\text { comunidade foram associados a um menor risco de } \\
\text { sofrer abuso. }\end{array}$ & $\begin{array}{l}\text { O fato da confiança na comunidade diminuir riscos deve } \\
\text { ser levado em consideração ao desenvolver estratégias de } \\
\text { base populacional para prevenir o abuso de idosos. }\end{array}$ \\
\hline
\end{tabular}




\begin{tabular}{|c|c|c|c|}
\hline Autor/ano & Objetivos & Resultado & Conclusão \\
\hline $\begin{array}{l}\text { Santos MAB, et al. } \\
(2020) .\end{array}$ & $\begin{array}{l}\text { Realizar uma revisão sistemática da } \\
\text { literatura de estudos epidemiológicos } \\
\text { analíticos sobre os fatores associados à } \\
\text { violência contra idosos. }\end{array}$ & $\begin{array}{l}\text { Os fatores associados à violência geral foram idade, } \\
\text { sexo, estado civil, nível de educação, renda, arranjo } \\
\text { familiar, suporte social, solidão, transtorno mental, } \\
\text { depressão, tentativa de suicídio, dependência para } \\
\text { atividades da vida diária, função cognitiva, doenças } \\
\text { crônicas, abuso de álcool ou drogas, entre outros. }\end{array}$ & $\begin{array}{l}\text { A VCPI é um fenômeno multifatorial e complexo, por isso } \\
\text { não pode ser vista de forma parcial, unidimensional e sim } \\
\text { levando em consideração todas as dimensões e entender } \\
\text { que há uma interdependência entre elas. }\end{array}$ \\
\hline $\begin{array}{l}\text { Chen W, et al. } \\
\text { (2019). }\end{array}$ & $\begin{array}{l}\text { Explorar a relação entre traços de } \\
\text { personalidade, características de } \\
\text { cuidado e tendência de abuso entre } \\
\text { cuidadores profissionais de idosos com } \\
\text { demência em instituições de longa } \\
\text { permanência na China. }\end{array}$ & $\begin{array}{l}\text { Os fatores protetores da tendência ao abuso foram a } \\
\text { amabilidade dos cuidadores, a fonte de finanças dos } \\
\text { destinatários dos cuidados e a duração da demência, } \\
\text { enquanto a maior dificuldade de atendimento e a } \\
\text { presença de sintomas comportamentais e psicológicos } \\
\text { de demência em idosos foram os fatores de risco. }\end{array}$ & $\begin{array}{l}\text { Traçar a personalidade dos cuidadores e as } \\
\text { características de cuidado de idosos com demência } \\
\text { podem ser relevantes para a tendência de abuso em } \\
\text { instituições de longa permanência. }\end{array}$ \\
\hline $\begin{array}{l}\text { Meyer K, et al. } \\
\text { (2019). }\end{array}$ & $\begin{array}{l}\text { Identificar abordagens que podem ser } \\
\text { testadas para prevenir maus-tratos a } \\
\text { idosos nas mãos de cuidadores familiars. }\end{array}$ & $\begin{array}{l}\text { Embora a VCPI seja distinta de outras áreas de violência } \\
\text { familiar, incluindo maus-tratos infantis e violência por } \\
\text { parceiro íntimo, fatores de risco comuns e fundamentos } \\
\text { teóricos apontam para oportunidades para estratégias de } \\
\text { prevenção. }\end{array}$ & $\begin{array}{l}\text { Estratégias de prevenção primária baseadas em } \\
\text { evidências de outros campos da violência familiar } \\
\text { oferecem um ponto de partida útil para intervenções para } \\
\text { prevenir VCPI. }\end{array}$ \\
\hline $\begin{array}{c}\text { Kulakçı AH e } \\
\text { Korkmaz AG (2019). }\end{array}$ & $\begin{array}{l}\text { Determinar a prevalência de abuso de } \\
\text { idosos entre idosos da comunidade e } \\
\text { seus fatores de risco associados. }\end{array}$ & $\begin{array}{l}\text { Os fatores de risco para abuso foram idade igual ou } \\
\text { superior a } 75 \text { anos, ser analfabeto, falta de renda propria, } \\
\text { não ter casa propria e ter relações familiars ruins. }\end{array}$ & $\begin{array}{l}\text { os, principalmente aqueles com } 7 \\
\text { ixa escolaridade, sem casa próp } \\
\text { devem ser avaliados quanto a ma }\end{array}$ \\
\hline $\begin{array}{c}\text { Alexa ID, et al. } \\
(2019) .\end{array}$ & $\begin{array}{l}\text { Explorar a VCPI em uma populaão } \\
\text { hospitalizada. }\end{array}$ & $\begin{array}{l}\text { Os pacientes abusados tinham uma função cognitiva } \\
\text { prejudicada. Eles também estavam desnutridos e } \\
\text { deprimidos. A presença de doença arterial periférica, } \\
\text { acidente vascular cerebral, pneumonia, doença renal } \\
\text { crônica, doenças musculoesqueléticas e ansiedade } \\
\text { correlacionaram-se com a presença de abuso. }\end{array}$ & $\begin{array}{l}\text { Ferramentas para detectar o abuso de idosos devem ser } \\
\text { incluídas na avaliação geriátrica padrão para prevenir o } \\
\text { envelhecimento. O número de idosos abusados é } \\
\text { significativo e os múltiplos fatores associados ao abuso } \\
\text { são diversos. }\end{array}$ \\
\hline Fang B, et al. (2019). & $\begin{array}{l}\text { Identificar fatores de risco e proteção } \\
\text { associados ao abuso de idosos entre } \\
\text { chineses idosos com deficiência } \\
\text { cognitiva e física na República Popular } \\
\text { da China. }\end{array}$ & $\begin{array}{l}\text { Sobrecarga do cuidador, sintomas neuropsiquiátricos do } \\
\text { receptor de cuidados e deficiências cognitivas são } \\
\text { fatores de risco proeminentes para o abuso com pessoas } \\
\text { idosas. }\end{array}$ & $\begin{array}{l}\text { Fatores semelhantes e distintos foram observados para } \\
\text { diferentes formas de abuso, potencialmente úteis para a } \\
\text { elaboração de programas de prevenção e intervenção. }\end{array}$ \\
\hline $\begin{array}{l}\text { Santos AMRD, et al. } \\
\text { (2019). }\end{array}$ & $\begin{array}{l}\text { Analisar as evidências disponíveis na } \\
\text { literatura de enfermagem acerca da } \\
\text { violência financeiro-patrimonial contra } \\
\text { idosos. }\end{array}$ & $\begin{array}{l}\mathrm{Na} \text { análise temática, emergiram três categorias: dados } \\
\text { epidemiológicos, fatores de risco e instituições } \\
\text { provedoras de serviços aos idosos. Verificou-se a } \\
\text { ocorrência deste tipo de violência de forma } \\
\text { concomitante. }\end{array}$ & $\begin{array}{l}\text { Verificou-se importantes dados epidemiológicos, fatores } \\
\text { de risco e características desse tipo de violência, inclusive } \\
\text { dentro das instituições prestadoras de serviço a idosos, } \\
\text { em diferentes países, aspectos essenciais para estruturar } \\
\text { e repensar políticas públicas de proteção e valorização da } \\
\text { pessoa idosa. }\end{array}$ \\
\hline
\end{tabular}

Fonte: Santos GSNF, et al., 2021. 
Sabe-se ainda que, entre os 41 artigos pré-selecionados, quanto ao idioma, os artigos em inglês predominaram, com $80,5 \%$. Artigos em português corresponderam a $19,5 \%$, e nenhum artigo em espanhol foi selecionado.

No que tange ao ano de publicação, constatou-se maior prevalência no ano de 2019 , com treze $(31,8 \%)$ artigos, seguido por 2017, 2020 e 2018, com onze (26,8\%), oito (19,5\%) e sete artigos (17\%), respectivamente. Do ano de 2021 houve dois artigos (4,9\%).

Observou-se também que houve predomínio da base de dados MEDLINE sobre LILACS e BDENF, onde foram encontrados trinta e quatro (83\%) artigos componentes da amostra na primeira base e sete (17\%) artigos na segunda, respectivamente. Dos sete estudos da LILACS, dois (4,9\% da amostra total) encontravam-se também na BDENF.

Finalmente, após a leitura na íntegra e com o instrumento pronto, foram extraídos argumentos dos artigos selecionados, comparando-os e agrupando-os por similaridade de conteúdo, sendo dessa maneira construída uma categoria para análise.

\section{DISCUSSÃo}

Com a finalidade de elaborarmos uma discussão acerca dos artigos selecionados, mostrou-se relevante a categoria: Violência Contra a Pessoa Idosa (VCPI) seus fatores de risco predominantes no cenário atual e estratégias adotadas para sua prevenção, que desvelou sobre os fatores de risco predominantes no cenário atual, assim como enfocou as estratégias adotadas para a prevenção a tal problemática.

Tendo em consideração as mudanças sociais, Sooryanarayana R, et al. (2017) apontam que a velha cultura de valores está sendo diluída na sociedade. Idoso foi por muito tempo sinônimo de respeito; porém, hoje nota-se que essa credibilidade vem perdendo um pouco o sentido para algumas pessoas e essa degradação da cultura de valores está ocorrendo junto com as mudanças na estrutura da família, urbanização e modernização (SOORYANARAYANA R, et al., 2017).

Dessa forma, através da literatura ficou nítido alguns fatores de risco associados a violência contra a pessoa idosa, como dependência funcional, deficiência cognitiva, baixo nível socioeconômico, idade, dependência financeira, gênero feminino, idade mais avançada, raça e etnia (VAN DEN BRUELE AB, et al., 2019; CHOI YJ, et al., 2018; KULAKÇI AH e KORKMAZ AG, 2019).

Em consonância, outros estudos, apontam como preditores como dependência funcional para as atividades de vida diária, diminuição na função cognitiva, idade, sexo feminino, estado civil, baixo nível de educação, renda familiar baixa, arranjo familiar, suporte social, solidão, transtorno mental, depressão, tentativa de suicídio, doenças crônicas, abuso de álcool ou drogas, entre outros (YADAV UN, et al., 2018; FILIPSKA K, et al., 2020; SANTOS MAB, et al., 2020). Nesse enfoque, Guruge S, et al. (2019), desenvolveram um processo de categorização para tais fatores de risco, em diferentes níveis: aqueles que estavam ligados à vítima, os relacionados ao abusador, a relação da vítima com o agressor, o da comunidade e da sociedade.

No tocante ao indivíduo vítima, foi citado a dependência emocional, a deficiência física e saúde mental. Em relação à comunidade, foi visto que a localização geográfica é um ponto importante, assim como as normas sociais e culturais da sociedade são elementos fundamentais no que se refere à exposição dos idosos a riscos. Outros trabalhos, de modo consonante ressaltaram que a ansiedade, depressão, transtorno de estresse pós-traumático, baixa autoestima, baixa auto-competência e satisfação com a vida, além da ideação suicida, como possíveis fatores de risco para o abuso de idosos (SPIRA M, et al., 2020; ANTEQUERA IG, et al., 2021; KOGA C, et al., 2020; SANTOS AMRD, et al., 2019). Burnes D, et al. (2017) em sua pesquisa relataram que indivíduos mais jovens têm maiores chances de sofrerem com casos mais graves de abuso emocional, físico e negligência.

Em contrapartida, estudo realizado no âmbito nacional, apontou que características sociodemográficas, como a idade, o sexo e a situação conjugal, não representam fatores de risco para a violência contra pessoas idosas, uma vez que estes não foram identificados como determinantes para o aumento de maus-tratos aos 
idosos na referida pesquisa, salientando-se que, quanto mais longevo, maior a vulnerabilidade à violência, indo em confronto a outra pesquisa desenvolvida em cenário internacional (PIRI N, et al., 2018; BOWS H, 2017). Entretanto, a autopercepção da saúde revela-se como um fator significativo, e quanto pior é essa percepção, mais provável é a ocorrência dos maus-tratos em consonância com outro estudo (BARROS RLDM, et al., 2019; GURSOY MY e KARA F, 2019)

Também com o intuito de identificar os fatores de risco associados a violência aos idosos no Brasil, Blay SL, et al. (2017), observou fatores como falta de educação, desemprego, pouca atividade física, doenças cardiovasculares e distúrbios psiquiátricos, associados ao abuso à pessoa idosa. Outra pesquisa, realizada em Cingapura, constatou como fatores, o ambiente familiar hostil, atrelados a abusos físicos e psicológicos. Além disso, os sentimentos de solidão também aumentavam o risco de abusos (CHOKKANATHAN S, 2018; WANG F, et al., 2018). Nesse contexto, uma pesquisa evidenciou que a maior parte dos cuidadores que agridem o idoso, provavelmente apresentam algum problema de ordem cognitiva/mental e/ou físico (Nobels A, et al., 2020).

Nessa perspectiva, pesquisadores demonstraram que a relação de suporte da pessoa idosas com cônjuge, familiares e amigos, emergiu como um fator positivo frente a ocorrência de violências, haja vista que o suporte social negativo aumenta a propensão do idoso a ser vítima de maus-tratos (ZHENG S, et al., 2019). Outra pesquisa, revisou a epidemiologia, fatores de risco e intervenções usadas para o abuso de idosos na Diáspora Africana. Tal estudo avaliou a população de diversos países de origem africana, desde o próprio continente da África Subsaariana, Caribe até mesmo nos Estados Unidos da América. Os resultados epidemiológicos variaram de acordo com a população avaliada, com uma prevalência de $24,9 \%$ a $81,1 \%$. Os fatores de risco associados são deficiência cognitiva e física, isolamento social, baixo poder econômico e viuvez (MOUTON CP e SOUTHERLAND JH, 2017).

De acordo com Clarysse $\mathrm{K}$, et al. (2018), os fatores de risco para o abuso de idosos são tanto por origem sociodemográfica, como fatores próprios da vítima ou dos seus cuidadores. O estresse intrafamiliar é um fator de risco relevante, além deste, os fatores condicionados ao idoso são comprometimento cognitivo, gênero feminino, idade acima de 80 anos, incapacidade funcional, raça não branca, baixo nível socioeconômico, isolamento social e situação de vida compartilhada. Quanto aos fatores vinculados aos cuidadores, a situação de vida compartilhada, abuso de álcool ou outras substâncias, alto nível de estresse, dependência financeira ou emocional à pessoa idosa, carga de trabalho excessiva, falta de treinamento e preparo para atuar como cuidador, história de violência, problemas de saúde mental (CLARYSSE K, et al., 2018).

Estudo realizado na Espanha, apontou variáveis sociodemográficas, cognitivas e estado de dependência da vítima, ansiedade, depressão e sobrecarga do cuidador, como fatores de risco para a ocorrência de VCPI (ORFILA F, et al., 2018). Nesse enfoque, pesquisadores afirmam ainda, que o estado de saúde física do cuidador, a carga de cuidados e uso de substâncias são fatores relevantes associados ao abuso físico contra a pessoa idosa (FANG B, et al., 2019). No cenário nacional, uma pesquisa mencionou a depressão na pessoa idosa, como fator de risco para sofrer violência. Em relação aos cuidadores, observou-se elevados níveis de sobrecarga e problemas com álcool como preditores do ato violento (CHEN W, et al., 2019).

Chen W, et al. (2019) também avaliaram, a partir de questionários e escores, as características e tendência dos cuidadores de idosos com demência a serem agressivos e violentos contra os mesmos. Isso foi feito a partir de um estudo com 156 cuidadores de pessoas idosas com demência, abrigados em Instituições de Longa Permanência (ILPs) em Guangzhou, China. Nesse artigo foi demonstrado que existe uma relação significativa entre o estado psicossocial dos cuidadores ou as dificuldades de cuidado encontradas no local de instalação do idoso e a tendência de abuso aos idosos, por parte de seus cuidadores das ILPs.

Dois dos artigos abordam, ainda, algumas mudanças nos fatores de risco para à violência contra idosos durante a pandemia da COVID-19. O Sars-Cov-2, agente etiológico da doença, está limitando o contato físico entre pessoas idosas e seus cuidadores desde março de 2020, quando foi instituído o distanciamento social, além de desencadear medo, ansiedade, perda de emprego, tensões financeiras, redução de serviços sociais, sobrecarga do cuidado, abuso de substâncias por parte do cuidador. Tudo isso influencia diretamente no 
aumento dos maus-tratos contra idosos durante a pandemia (MAKAROUN LK, et al., 2021). Makaroun LK, et al. (2020) afirmam que as limitações de contato e o isolamento social, consequentes da pandemia da COVID19, suprimem o rastreamento e as intervenções por parte de serviços de proteção das pessoas da terceira idade, o que dificulta a percepção dos maus-tratos e, consequentemente, interfere no aumento dos riscos e dos casos de violência contra as pessoas idosas.

Um estudo produzido por Carmona-Torres JM, et al. (2017), nas Ilhas dos Açores, tinha como objetivos dimensionar os maus-tratos contra as pessoas idosas, vulneráveis no ambiente familiar e comunitário, identificando fatores de risco para maus-tratos e definindo o perfil do idoso maltratado. Demonstrou-se que $24,5 \%$ dos idosos participantes possuíam suspeita de maus-tratos, desses $46,66 \%$ relataram sofrer abuso psicológico, $30 \%$ sofriam com negligência, $13,33 \%$ abuso econômico e $10 \%$ violência física. Em $69,54 \%$ dos casos o agressor era um parente de primeiro grau, no qual os filhos aparecem como os principais responsáveis pela violência $(43,45 \%)$. Foram encontrados como fatores de risco para os abusos, ser do sexo feminino e pertencer a uma família hostil.

Outro artigo foi realizado com o objetivo de identificar os fatores de risco e a prevalência de violência contra idosos cometida pelos parceiros íntimos destes. Verificou-se que a violência por parceiro íntimo acometeu tanto mulheres quanto homens idosos, sendo a violência psicológica e financeira os tipos mais comuns. Os principais fatores de risco associados foram comprometimento funcional, depressão, baixa renda, exposição pregressa à violência e consumo de álcool (WARMLING D, et al., 2017).

Uma outra pesquisa realizada nos Estados Unidos, investigou-se a ocorrência dos tipos de violência mais prevalentes, demonstrando-se que viver nos Estados Unidos há mais tempo, denotou um maior risco de exploração financeira e falar mandarim ou inglês apresentou maior risco de sofrer violência sexual ou física (DONG X, et al., 2017).

No quesito de doenças psiquiátricas, Sudan A, et al. (2020) avaliaram através de uma ferramenta de triagem a prevalência do abuso nos pacientes que se enquadravam nessa categoria e identificaram um aumento significativo da violência naqueles que sofriam com algum distúrbio, quando comparados aqueles sem patologias psicológicas. Ainda apontaram uma forte correlação do abuso com o gênero, alfabetização e estado civil. A vista disso, é notório que se torna um ciclo vicioso, em que o idoso sente medo, solidão, sensação de abandono e acaba agravando a sua doença psiquiátrica.

Já a ocorrência de algumas patologias como Acidente vascular encefálico, pneumonia, doença renal crônica, ansiedade e doenças musculoesqueléticas, também apresentam relação com o desenvolvimento do abuso contra a pessoa idosa (ALEXA ID, et al., 2019; MOHSENI, M, et al. 2019)). Um estudo no âmbito internacional, apontou a deficiência física como um fator comum para a prática de abuso contra o idoso, haja vista que tal fator corrobora para o aumento da dependência de cuidados por parte destes (SEMBIAH S, et al., 2020).

No tocante às estratégias adotadas para a prevenção da VCPI, ressalta-se que a prevenção primária pode ser considerada uma importante ferramenta na intervenção da violência contra pessoas idosas, como por exemplo, a prática da visita domiciliar, capaz de detectar idosos vulneráveis, sob responsabilidade de um cuidador, com riscos à ocorrência de maus-tratos (MEYER K, et al., 2019).

Outra estratégia de extrema importância é a utilização da Caregiver Abuse Screen (CASE), instrumento que detecta o potencial abusivo dos cuidadores de idosos. Por meio da utilização da CASE, é possível criar estratégias de prevenção a ocorrência de VCPI (MELCHIORRE MG, et al., 2017).

Day A, et al. (2017) produziu um estudo após a análise de artigos de revisões sistemáticas sobre os resultados de medidas de prevenção a violência aos idosos, com o objetivo de reconhecer a potencialidade dessas medidas. Entretanto, houve grande falta de informações sobre os resultados em determinados artigos analisados e isso mostrou que a eficácia das possíveis intervenções estudadas é limitada não apenas pela pequena quantidade de estudos realizados, mas também pela baixa qualidade deles. Das medidas identificadas, apenas duas obtiveram um projeto de avaliação esperado para determinar a relação causal entre a intervenção e a redução do risco. 
Dantas RB, et al. (2017) adaptaram e avaliaram as propriedades psicométricas da Escala Triagem de Vulnerabilidade ao Abuso (Vulnerability to Abuse Screening Scale, VASS) em uma amostra de 151 idosos com idade média de 72,05 anos, mínima de 60 anos e máxima de 92 anos, da cidade de Belo Horizonte, com o propósito de reconhecer o risco de violência doméstica contra essa população no Brasil. Assim, obteve resultados aceitáveis em alguns quesitos, como o da dimensionalidade, mas apresentou limitações na testagem da sensibilidade e especificidade do instrumento de triagem.

\section{CONSIDERAÇÕES FINAIS}

A face do exposto, é possível perceber que com a transição demográfica e o envelhecimento populacional, também cresce a VCPI, trazendo significativas implicações sociais. Por isso, compreender os fatores de risco que afetam a vida desse grupo populacional é crucial para definir estratégias de prevenção direcionadas. Desse modo, no que se refere aos fatores de risco, foi identificado que o sexo, a idade, o estado civil, o meio intrafamiliar, a presença de deficiência física e/ou cognitiva, o poder econômico e a sobrecarga do cuidador são elementos que podem influenciar diretamente no abuso contra idosos. Sob o contexto da prevenção, foi constatado interferência de apoio social, independência financeira e envelhecimento ativo. Entretanto, muito ainda precisa ser estudado e publicado acerca dos mecanismos para prevenir os fatores de riscos VCPI.

\section{REFERÊNCIAS}

1. ALEXA ID, et al. Elder abuse and associated factors in eastern romania. Psychogeriatrics, 2019; 20(2): $196-205$.

2. ANTEQUERA IG, et al. Rastreamento de violência contra pessoas idosas: associação com estresse percebido e sintomas depressivos em idosos hospitalizados. Esc. Anna Nery Rev. Enferm., 2021; 25(2): 1-8.

3. BARCELOS EM, MADUREIRA MDS. Violência contra o idoso. In: Chaimowicz F, organizador. 2. ed. Belo Horizonte: Nescon UFMG; 2013. p. 138-149

4. BARROS RLDM, et al. Violência doméstica contra idosos assistidos na atenção básica. Saúde em Debate, 2019; 43(122): 793-804.

5. BURNES D, et al. Elder abuse severity: A critical but understudied dimension of victimization for clinicians and researchers. The Gerontologist, 2017; 57(4): 745-756.

6. CARMONA-TORRES JM, et al. Maus-tratos no ambiente familiar contra idosos nas Ilhas dos Açores. Revista LatinoAmericana de Enfermagem, 2017; 25.

7. CHEN W, et al. The relationship between personality traits, caring characteristics and abuse tendency among professional caregivers of older people with dementia in long-term care facilities. Journal of clinical nursing, 2019.

8. CHOI YJ, et al. Associations among Elder Abuse, Depression and PTSD in South Korean Older Adults. Int. J. Environ. Res. Public Health, 2018.

9. CHOKKANATHAN S. Prevalência e correlatos de maus-tratos a idosos em Cingapura. Jornal de abuso e negligência de idosos, 2018; 30 (4): 271-283.

10. CLARYSSE K, et al. Sinais de abuso físico e negligência no paciente maduro. Clinics in dermatology, $2018 ; 36$ (2): 264-270.

11. DANTAS RB, et al. Propriedades psicométricas da Vulnerability to Abuse Screening Scale para rastreio de abuso contra idosos. Revista de Saúde Pública, 2017; 51 (31): 1-11

12. DAY A, et al. An assessment of interventions that target risk factors for elder abuse. Health and Social Care in the Community, 2017; 25(5): 1532-1541.

13. DONG $X$, WANG B. Incidence of elder abuse in a US Chinese population: Findings from the longitudinal cohort PINE study. Journals of Gerontology Series A: Biomedical Sciences and Medical Sciences, 2017 ; 72 (1): 95-101.

14. FANG B, et al. Risk and protective factors associated with domestic abuse among older Chinese in the People's Republic of China. Archives of gerontology and geriatrics, 2019; 82: 120-127.

15. FILIPSKA K, et al. Prevalence and associated factors of elder psychological abuse-a cross-sectional screening study, based on a hospitalized community from Poland. Archives of gerontology and geriatrics, 2020; 90: 1-7.

16. GURSOY MY, KARA F. Prevalence of violence against older adults and associated factors in Çanakkale, Turkey: A cross-sectional study. Geriatrics \& gerontology international, 2019; 20(1): 66-71.

17. GURUGE S, et al. Developing a comprehensive understanding of elder abuse prevention in immigrant communities: a comparative mixed methods study protocol. BMJ open, 2019; 9(1): 1-6.

18. INSTITUTO BRASILEIRO DE GEOGRAFIA E ESTATÍSTICA (IBGE). Síntese de indicadores sociais: Uma análise das condições de vida. Rio de Janeiro: IBGE; 2015.2 Disponível em: https://biblioteca.ibge.gov.br/visualizacao/livros/liv95011.pdf. Acesso em: 26 de maio de 2021. 
19. KOGA C, et al. Elder abuse and social capital in older adults: The Japan Gerontological Evaluation Study. Gerontology, 2020; 66(2): 149-159.

20. KULAKÇI AH, KORKMAZ AG. Prevalence of elder abuse among community-dwelling older adults in Turkey and its associated factors. Psychogeriatrics, 2019; 20(1): 3-10.

21. MAKAROUN LK, et al. Changes in Elder Abuse Risk Factors Reported by Caregivers of Older Adults During the COVID-19 Pandemic. Journal of the American Geriatrics Society, 2021; 69 (3): 602-603.

22. MAKAROUN LK, et al. Elder abuse in the time of COVID-19: Increased risks for older adults and their caregivers. The American Journal of Geriatric Psychiatry, 2020; 28(8): 876- 879.

23. MEYER K, et al. What can elder mistreatment researchers learn about primary prevention from family violence intervention models?. The Gerontologist, 2019; 59(4): 601-609.

24. MOHSENI M, et al. Prevalence of elder abuse and associated factors among community-dwelling older adults in Iran. Journal of elder abuse \& neglect, 2019; 1-10.

25. MOURA LKB, et al. Análise bibliométrica das evidências científicas sobre violência contra a pessoa idosa. Ciênc. saúde coletiva, 2020; 25(6): 2143-2152.

26. NOBELS A, et al. "Too Grey To Be True?" Sexual Violence in Older Adults: A Critical Interpretive Synthesis of Evidence. International journal of environmental research and public health, 2020; 17(11): 1 -12.

27. SANTOS AMRD, et al. Violência financeiro-patrimonial contra idosos: revisão integrativa. Revista Brasileira de Enfermagem, 2019; 72: 328-336.

28. SANTOS MAB, et al. Fatores associados à violência contra o idoso: uma revisão sistemática da literatura. Ciênc. Saúde Coletiva, 2020; 25(6): 2153-2175.

29. SEMBIAH S, et al. Elder abuse and its predictors: a cross-sectional study in a rural area of West Bengal, eastern part of India. Psychogeriatrics, 2020; 20(5): 636-644.

30. SOUZA MTD, et al. Revisão integrativa: o que é e como fazer. Einstein (São Paulo), 2010; 8(1): 102-106.

31. SPIRA M, et al. Trauma from physical and emotional sibling violence as a potential risk factor for elder abuse. Journal of gerontological social work, 2020; 63(3): 162-173.

32. SUDAN A, et al. Prevalence of Abuse in Elders With Psychiatric Morbidity and Its Sociodemographic Association. Cureus, 2020; 12(4): 1-6.

33. UNITED NATIONS. Department of Economic and Social Affairs, Population Division (2017). World Population Prospects. $2017 . \quad$ Disponível em: https://www.un.org/development/desa/pd/sites/www.un.org.development.desa.pd/files/files/documents/2020/Jan/un_ 2017_world_population_prospects-2017_revision_databooklet.pdf. Acesso em: 25 de maio de 2021.

34. VAN DEN BRUELE AB, et al. Elder Abuse. Clin. Geriatr. Med., 2019; 35: 103-113.

35. VASCONCELOS AMN, GOMES MMF. Transição demográfica: a experiência brasileira. Epidemiol. Serv. Saúde, 2012; 21(4): 539-548

36. WANG F, et al. Elder abuse and its impact on quality of life in nursing homes in China. Archives of gerontology and geriatrics, 2018; 78: 155-159.

37. YADAV UN, et al. The time has come to eliminate the gaps in the under-recognized burden of Elder mistreatment: $A$ community-based, crosssectional study from rural eastern Nepal. PLoS ONE, 2018; 13(6).

38. ZHENG S, et al. Sources and variations in social support and risk for elder mistreatment in a US Chinese population. Journal of the American Geriatrics Society, 2019; 67(3): 499-505. 